\title{
Regional hyperthermia of the abdomen, a pilot study towards the treatment of peritoneal carcinomatosis
}

Marcus Beck ${ }^{1 *}$, Pirus Ghadjar ${ }^{1}$, Mirko Weihrauch?', Susen Burock², Volker Budach', Jacek Nadobny', Jalid Sehouli ${ }^{3}$ and Peter Wust ${ }^{1 *}$

\begin{abstract}
Background: Peritoneal carcinomatosis occurs in different cancer subtypes and is associated with a dismal prognosis. Some doubts remain whether the whole abdomen can be treated by regional hyperthermia, therefore we analyzed feasibility conducting a pilot study.

Methods: A simulation of the abdominopelvic heat distribution in 11 patients with peritoneal carcinomatosis was done using the HyperPlan software and the SIGMA-60 and SIGMA-Eye applicators. Tissue-specific region-related electrical and thermal parameters were used to solve the Maxwell's equations and the bioheat-transfer equation. Three-dimensional specific absorption rate (SAR) distributions and, additionally, estimated region-related perfusion rates were used to solve the bioheat-transfer equation. The predicted SAR and temperature distributions were compared with minimally invasive measurements in pelvic reference points.

Results: In 11 patients (7 of them treated in the SIGMA-60 and 4 in the SIGMA-Eye applicator) the measured treatment variables (SAR, temperatures in the pelvic reference points) indicated that the heated volumes were higher for the SIGMA-Eye applicator. The mean computed abdominal SARs were less for the SIGMA-Eye (33 versus $44 \mathrm{~W} / \mathrm{kg}$ ). Nevertheless, the temperature distributions in the abdomen (peritoneal cavity) were more homogeneous in the SIGMA-Eye applicator as compared to the SIGMA-60 as indicated by higher values of $T_{90}$ (mean 40.2 versus $38.2^{\circ} \mathrm{C}$ ) and $\mathrm{T}_{50}$ (mean 41.1 versus $40.2^{\circ} \mathrm{C}$ ), while the maximum temperatures were similar (in the range 41 to $43^{\circ} \mathrm{C}$ ). Even though the mean abdominal SAR was lower in the SIGMA-Eye, the heat distribution covered a larger volume of the abdomen (in particular the upper abdomen).

For the SIGMA-60 applicator the achieved $T_{90}$ appeared to be limited between 41 and $42{ }^{\circ} \mathrm{C}$, for the SIGMA Eye applicator more effective $T_{90}$ in the range 42 to $43{ }^{\circ} \mathrm{C}$ were obtained.

Conclusion: Our results suggest that an adequate heating of the abdomen and therefore abdominal regional hyperthermia in PC patients appears feasible. The SIGMA-Eye applicator appears to be superior compared to the SIGMA-60 applicator for abdominal hyperthermia.
\end{abstract}

Keywords: Hyperthermia, Heat, Cancer, Peritoneal carcinosis, Heatability

\footnotetext{
*Correspondence: marcus.beck@charite.de; peter.wust@charite.de

'Department of Radiation Oncology, Charité Universitätsmedizin Berlin,

Augustenburger Platz 1, 13353 Berlin, Germany

Full list of author information is available at the end of the article
} 


\section{Introduction}

Peritoneal carcinomatosis (PC) occurs in different cancer subtypes and is associated with a dismal prognosis. In non-gynecologic malignancies such as colorectal, gastric, pancreatic cancer as well as in peritoneal mesothelioma, pseudomyxoma peritonei and primary peritoneal carcinoma a median overall survival (OS) of 3,1 months was reported $[1,2]$.

On the other hand, ovarian cancer is the most frequently type of gynecologic cancer accompanied by PC. In a single center analysis of 214 patients with primary ovarian cancer the initial surgery detected a peritoneal manifestation in $76 \%$ [3]. The 5 year OS in advanced stage ovarian cancer (Stage III/IV) is approximately only $25 \%$ [4].

During the last decades several efforts have been made to improve the therapy of PC. Besides intravenous chemotherapy and cytoreductive surgery (CRS), the intraperitoneal chemotherapy and lastly CRS followed by hyperthermic intraperitoneal chemotherapy (HIPEC) were implemented. CRS and HIPEC were claimed to be beneficial for patients with pseudomyxoma peritonei, peritoneal mesothelioma and colorectal cancer [5, 6]. Less is known regarding the role of CRS and HIPEC for $\mathrm{PC}$ in gastric cancer and for ovarian cancer [7-11]. As a general limitation CRS is associated with notable morbidity, mortality and a restriction of suitable patients [7-11]. Therefore abdominal regional hyperthermia (RHT) combined with intravenous chemotherapy appears to be an important non-invasive and well tolerated option in the treatment of PC [12]. In two phase I/II studies at our center we demonstrated that this approach was associated with encouraging OS rates and low RHT associated toxicities in patients suffering from PC of colorectal and ovarian cancers $[13,14]$. However, the beneficial effect of RHT for the treatment of PC has never been demonstrated in a randomized trial. Additionally, there were some doubts whether RHT, using commercially available applicators (such as the SIGMA-60 and SIGMA-Eye), could actually lead to an appropriate heat distribution covering the whole abdomen (including the peritoneal cavity). To facilitate the future use of RHT for the treatment of PC as well as the need for innovative randomized trials incorporating RHT we performed a planning study to evaluate whether RHT of the abdomen using SIGMA-applicators is feasible.

\section{Methods}

We generated patient models for a group of 11 treated patients with PC. These 11 patients suffered from PC originating from different cancer subtypes and were randomly selected from a larger cohort which underwent RHT in the SIGMA-60 applicator or SIGMA-Eye applicator within a phase I/II trial, as previously reported [13, 14]. On computed tomography (CT) scans we segmented the target volume (abdominal cavitiy) and surrounding organs and structures like liver, kidneys, bones, muscles, fatty tissue, vagina and bladder. Than specific patient models with individual tetrahedron grids were generated and the finite elements method (FEM) was used for the model calculations. The specific absorption rate (SAR)-distributions $(\mathrm{W} / \mathrm{kg})$ were calculated using the treatment planning system HyperPlan as previously described $[15,16]$. For the simulations we used assumed electrical, thermal and physiological parameters of the tissues according to the values previously reported. See [17-19] and Table 1 . The parameters for permittivity and conductivity showed no relevant frequence dependend variety in our measurement (70-100 MHz) and so the value for $90 \mathrm{MHz}$ was used. These parameters in conjunction with predicted SAR distributions were used to solve the bioheat-transfer equation. Applicator models for the SIGMA-60 applicator (8 dipole antennas arranged in pairs and connected to 4 amplifier channels) and SIGMA-Eye applicator (24 dipole antennas arranged in pairs and connected to 12 amplifier channels) were available. The hyperthermia planning system HyperPlan has been tested and validated for both applicator models $[15,16]$.

The SAR and temperature distributions predicted via HyperPlan can be compared with measurements in single pelvic reference points in the rectum, vagina and bladder (marked with star symbols in Fig. 1) by using the point probe software tool of HyperPlan, which enables a measurement of SAR or temperature of every specified point. The measurement of SAR was performed using the temperature decline in the time-temperature curve after turning of the power after treatment.

Perfusion and its regulation during heat exposure is a crucial characteristic of the tissues in order to compute the temperature distribution. Liver and kidneys are the highest perfused organs. The perfusion of the abdomen was estimated as $20 \mathrm{ml} / 100 \mathrm{~g} / \mathrm{min}$ by assuming a mixture of the intestine (basal perfusion of $40-50 \mathrm{ml} / 100 \mathrm{~g} / \mathrm{min}$ ) and surrounding low perfused connective tissue. The

Table 1 Electrical parameters for computation of the power deposition patterns and perfusion parameters $(90 \mathrm{MHz})$

\begin{tabular}{llll}
\hline Tissue & $\mathcal{E}_{r}$ & $\sigma(\mathrm{S} / \mathrm{m})$ & Perfusion under RHT $(\mathrm{ml} / 100 \mathrm{~g} / \mathrm{min})$ \\
\hline Muscle & 80 & 0.8 & 30 \\
Fat & 10 & 0.04 & 20 \\
Abdomen & 36 & 0.55 & 20 \\
Liver & 78 & 0.6 & 100 \\
Kidneys & 83 & 1 & 400 \\
Rectum & 60 & 0.7 & 5 \\
Perirectal tissue & 23 & 0.3 & 20 \\
Vagina & 80 & 0.8 & 5 \\
Bladder & 80 & 0.6 & 5
\end{tabular}

$\mathcal{E}_{r}$ relative permittivity, $\sigma(\mathrm{S} / \mathrm{m})$ conductivity 


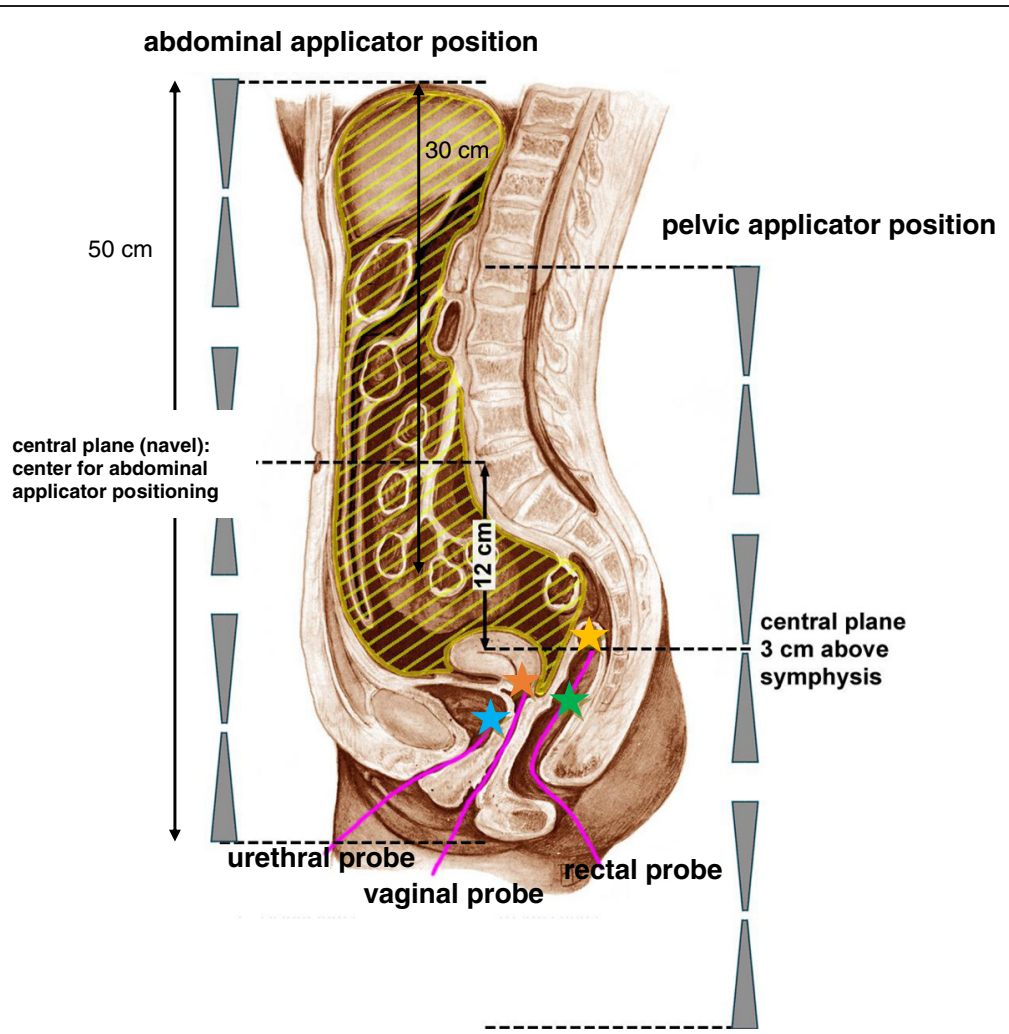

Fig. 1 Arrangement of the annular-phased-array applicator, centered on the navel, for abdominal heating and centered $3 \mathrm{~cm}$ above symphysis for pelvic heating. Sometimes the applicator position for SIGMA-60 differed from central plane as shown in Table 2. The reference points in endoluminal catheters are also depicted (marked with colored stars)

gastrointestinal tract has a large potential to increase perfusion during digestion (up to 6 fold) and has also (as other organs) a self-protection ability against temperature elevation. However, the thermoregulation in the visceral organs is less pronounced than in other tissues (e.g. skin) because the temperature in the portal vein might be as high as $40{ }^{\circ} \mathrm{C}$ under physiological conditions (caused by chemical processes during digestion). Therefore, a higher tolerance against temperature increase might be assumed in visceral organs. Furthermore, the intestinal perfusion might even be depressed under somatic stress such as regional hyperthermia. Therefore, $20 \mathrm{ml} / 100 \mathrm{~g} / \mathrm{min}$ is a reasonable approach to describe the peritoneal cavity [19]. The maximum temperature in the organs such as intestine, rectum and bladder was set to $43{ }^{\circ} \mathrm{C}$. The perfusion in other normal tissues such as muscle, fat or perirectal tissue is under hyperthermic conditions higher than the basal perfusion (Table 1).

In a first step, we reviewed for every patient, whether the measurements in the reference points (both SAR and temperature) could be correctly reproduced by the corresponding patient-specific plan (using HyperPlan). The computations are based on the patient anatomy (as basis for a patient model), the treatment variables (frequency, total power, phase amplitudes) and the tissue parameters according to Table 1 . We started with the applicator position described in the treatment protocol, but for matching the plan to the measured data a slight variation of the positions of the applicator and the point probes was admitted. Variations of the positions in relation to the navel central plane (Fig. 1) were listed in Table 2. Due to patient discomfort in some cases it was necessary to shift the SIGMA-60 applicator a few $\mathrm{cm}$ distal the navel as listed in Table 2.

In a second step, we evaluated the SAR and temperature distributions from HyperPlan in the abdomen. We used the described individual patient models, which were assumed as validated after successful matching in the first step.

In a third step, we varied (in particular increased) the total power in the available patient models to estimate the limitations of abdominal heating (with ring applicators) under favorable conditions. The total power was restricted, if a temperature threshold in any tissue was exceeded. Because the total power is increased in steps of $100 \mathrm{~W}$, such a maximum permissible temperature might be slightly passed in the simulation studies and the generated temperature plots. We assume a selfprotection of all healthy tissues by increasing the perfusion, if we go only slightly beyond the threshold. 
Table 2 Analysis of the SAR and temperature distribution in the abdomen

\begin{tabular}{|c|c|c|c|c|c|c|c|c|c|}
\hline SIGMA-60 & $\begin{array}{l}\text { SAR } 50 \text { (abdomen) } \\
\text { (W/kg) }\end{array}$ & $\mathrm{T}_{90}\left({ }^{\circ} \mathrm{C}\right)$ & $\mathrm{T}_{50}\left({ }^{\circ} \mathrm{C}\right)$ & $\mathrm{T}_{\max }\left({ }^{\circ} \mathrm{C}\right)$ & SIGMA-Eye & $\begin{array}{l}\text { SAR } 50 \text { (abdomen) } \\
\text { (W/kg) }\end{array}$ & $\mathrm{T}_{90}\left({ }^{\circ} \mathrm{C}\right)$ & $\mathrm{T}_{50}\left({ }^{\circ} \mathrm{C}\right)$ & $\mathrm{T}_{\max }\left({ }^{\circ} \mathrm{C}\right)$ \\
\hline $\begin{array}{l}70 \mathrm{MHz}, 700 \\
\mathrm{~W}_{1}-2 \mathrm{~cm}\end{array}$ & 45 & 38,3 & 40,1 & 41,8 & $\begin{array}{l}100 \mathrm{MHz} \\
1500 \mathrm{~W}\end{array}$ & 35 & 40,7 & 41,6 & 43,0 \\
\hline $\begin{array}{l}90 \mathrm{MHz}, 600 \\
\mathrm{~W},-3 \mathrm{~cm}\end{array}$ & 42 & 38,3 & 40,6 & 42,7 & $\begin{array}{l}100 \mathrm{MHz} \\
1600 \mathrm{~W}\end{array}$ & 36 & 39,9 & 41 & 42 \\
\hline $90 \mathrm{MHz}, 500 \mathrm{~W}$ & 42 & 38,1 & 39,5 & 41,2 & $\begin{array}{l}100 \mathrm{MHz} \\
1500 \mathrm{~W}\end{array}$ & 20 & 39,1 & 39,8 & 41,2 \\
\hline $90 \mathrm{MHz}, 600 \mathrm{~W}$ & 43 & 38,2 & 40,7 & 42,2 & $\begin{array}{l}100 \mathrm{MHz} \\
1500 \mathrm{~W}\end{array}$ & 40 & 41 & 41,9 & 42,8 \\
\hline $\begin{array}{l}90 \mathrm{MHz}, 500 \\
\mathrm{~W},-3 \mathrm{~cm}\end{array}$ & 52 & 39,1 & 41 & 43,7 & Mean value & 33 & 40,2 & 41,1 & 42,2 \\
\hline $\begin{array}{l}70 \mathrm{MHz}, 500 \\
\mathrm{~W},-4 \mathrm{~cm}\end{array}$ & 32 & 38 & 39,5 & 41,3 & & & & & \\
\hline $90 \mathrm{MHz}, 550 \mathrm{~W}$ & 40 & 37,6 & 39,8 & 42,2 & & & & & \\
\hline Mean value & 42 & 38,2 & 40,2 & 42,2 & & & & & \\
\hline
\end{tabular}

The applicator position is centered on the central plane (navel) as shown in Fig. 1. Distal aberrations (in cm) of applicator position to central position (required for a few patients treated with Sigma-60) are listed in the left column

SAR specific absorption rate

This analysis was conducted in accordance with the regulations of the local Ethics Committee of the Charite Universitätsmedizin Berlin.

\section{Results}

In 11 treated patients (7 of them in the SIGMA-60 and 4 in the SIGMA-Eye applicator) particular treatment variables (SAR, temperatures in the reference points) were determined. We selected a typical heating session for every patient. The average values for total power applied, absolute SAR and steady-state temperatures in the reference points for all patients on each applicator were created: For the SIGMA-60 applicator 500-700W, SAR(vagina) $22 \mathrm{~W} / \mathrm{kg}, \mathrm{SAR}$ (rectum) $16 \mathrm{~W} / \mathrm{kg}, \mathrm{T}_{\text {vagina }}$ $40,8{ }^{\circ} \mathrm{C}$ and $\mathrm{T}_{\text {rectum }} 40,3{ }^{\circ} \mathrm{C}$. For the SIGMA-Eye applicator 1400-1600W, SAR(vagina) $11 \mathrm{~W} / \mathrm{kg}, \mathrm{SAR}$ (rectum) $2 \mathrm{~W} / \mathrm{kg}, \mathrm{T}_{\text {vagina }} 40{ }^{\circ} \mathrm{C}$ and $\mathrm{T}_{\text {rectum }} 39,4{ }^{\circ} \mathrm{C}$. In all treatments the phase delays were 0 and amplitudes were equally weighted. No pattern optimization was performed, because we assumed that the balanced phase and amplitude control is near the optimum for the purpose of abdominal heating.

The measured values indicate that the heated volumes are higher for the SIGMA-Eye applicator, which needs therefore higher total power. Nevertheless, the mean SAR and temperatures in the pelvis are lower in the SIGMA Eye applicator in comparison to the SIGMA-60 applicator. However, the considerably higher total (amplifier) power required at the 12 amplifiers of the SIGMA-Eye is mainly due to a lower efficiency, which is nearly half of the SIGMA 60's efficiency. The lower efficiency is caused by losses in the matching networks (of short antennas) and feeding cables. Furthermore, this SIGMA-Eye applicator was used in a hybrid system where additional loss of efficiency is caused by specifics of the electro-technical separation of MRI and applicator [13]. All these attributes had been included into the applicator models used in HyperPlan. Note that the power levels given in this study for the SIGMA-Eye applicator are different from power levels in a SIGMA-Eye applicator outside the MRI.

We compared the measured temperatures and SAR in the reference points with the values in the corresponding patient-specific plans and performed a matching of all measurements simultaneously by adapting the applicator and point probe positions (see Methods). We found in all patients already a satisfactory agreement in the first approximation, but typically further improvement was possible by a straight forward search in an unambiguous direction of the parameter space. Finally, we achieved a fair correlation as depicted in Fig. 2 with a correlation coefficient of $r=0.997$ for SAR and $r=0.90$ for temperatures, respectively.

After completion of matching procedure we computed the SAR and the temperature distributions in the whole volume (pelvis and abdomen), using the verified patient models and the refined positions of the central plane (longitudinal shift from the belly button or navel). We achieved median SAR and index temperatures in these 11 patient models as summarized in Table 2.

We noted characteristic differences between both applicators according to Table 2. The mean SAR were less for the SIGMA-Eye (33 versus $44 \mathrm{~W} / \mathrm{kg}$ ). This was mainly due to the lower SAR in the pelvic region by using the SIGMA-Eye (see also Table 2). Nevertheless, the temperature distributions in the abdomen (peritoneal cavity) were more homogeneous in the SIGMA-Eye applicator, which was indicated by higher $\mathrm{T}_{90}(40.2$ 

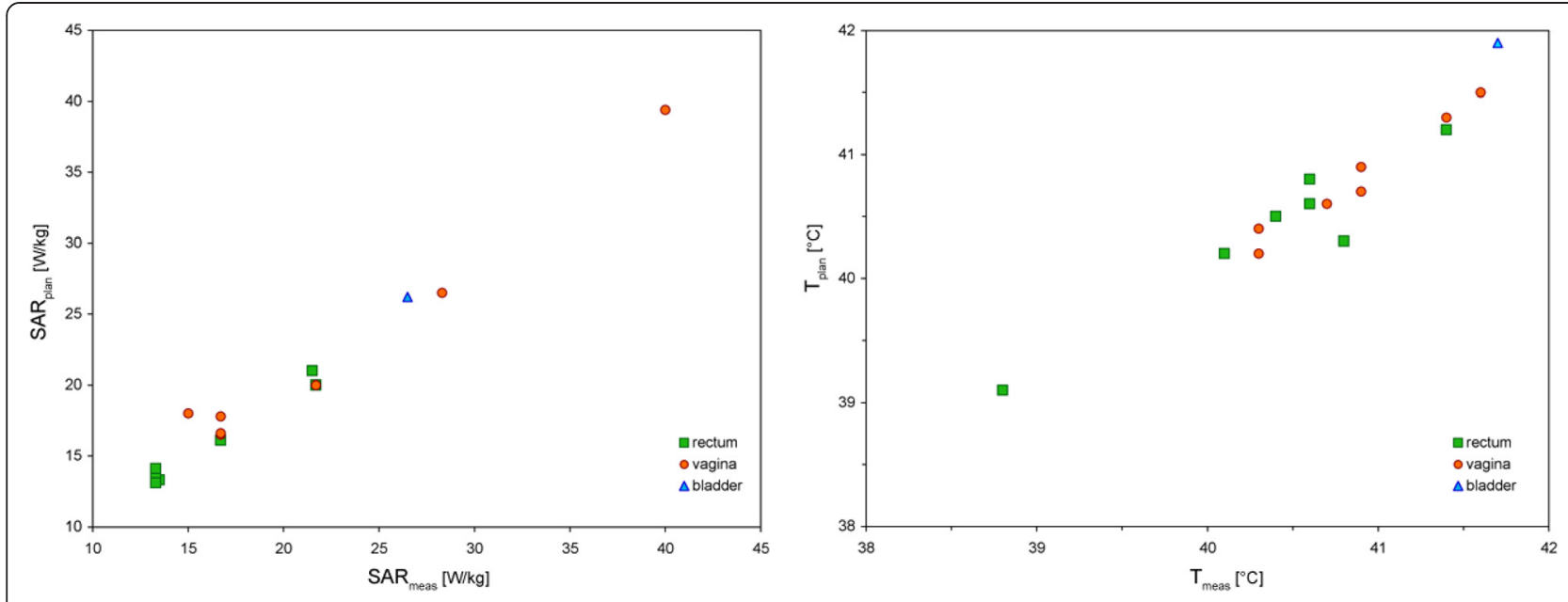

Fig. 2 Correlation of measured SAR in the reference points and calculated values in HyperPlan after a matching process (see left). Correlation of measured and calculated temperatures in the same reference points under the same conditions (see right). $r=0.997$ for SAR and $r=0.90$ for temperatures

versus $38.2{ }^{\circ} \mathrm{C}$ on average) and $\mathrm{T}_{50}\left(41.1\right.$ versus $40.2{ }^{\circ} \mathrm{C}$ on average), while the maximum temperatures were similar (in the range 41 to $43{ }^{\circ} \mathrm{C}$ ). Even though the mean SAR was lower in the SIGMA-Eye, the distribution covered a larger volume of the abdomen (in particular the upper abdomen). Therefore, the $\mathrm{T}_{90}$ for the SIGMA-Eye were above $40{ }^{\circ} \mathrm{C}$ on average, which might be sufficient for some sensitizing effect in conjunction with chemotherapy (and radiotherapy).

We proceeded with the simulation study by further increase of total power starting from the actual matched treatments with the SIGMA-60 or the SIGMA-Eye applicator. The $\mathrm{SAR}_{50}$ and $\mathrm{T}_{90}$ in the peritoneum belonging to a particular power level and applicator are determined plotted in a two-dimensional coordinate system (Fig. 3). The plotted points rearrange in characteristic lines corresponding to certain patients and applicators.

The total power was always turned off after a critical temperature was exceeded $\left(43{ }^{\circ} \mathrm{C}\right.$ in the organs). Under these conditions we could differentiate between easy-toheat (lines shifted to the left) and difficult-to-heat patients (lines shifted to the right). For the SIGMA-60 applicator the achieved $\mathrm{T}_{90}$ appeared limited between 41 and $42{ }^{\circ} \mathrm{C}$, and only exceptionally $\mathrm{T}_{90}$ reached $43{ }^{\circ} \mathrm{C}$. For the SIGMA Eye applicator we had to apply higher total power levels and obtained a more effective $\mathrm{T}_{90}$ in the range of 42 to $43{ }^{\circ} \mathrm{C}$. Even for the most difficult-to-heat patient we finally achieved a $\mathrm{T}_{90}$ of $42{ }^{\circ} \mathrm{C}$ without exceeding a temperature threshold. Evidently, we could heat a higher volume of the abdomen with more homogeneity and a better covering by use of the SIGMA Eye applicator.

The different patterns are illustrated by Fig. 4, which shows SAR and temperature distributions for both applicators under comparable conditions for a selected model. Both applicators were centered on the navel plane. The
SIGMA-60 applicator tended to heat the pelvis more effective. However, the SIGMA-Eye applicator covered the upper abdomen better and gained after all a better $T_{90}$ for the whole abdomen.

The pattern of the SIGMA-Eye applicator may even be improved by proper phase control. In our simulations we could show the effect of a delay on the outer rings of the applicator resulting in a broadening of the effective heating volume and an increased SAR. A phase delay of

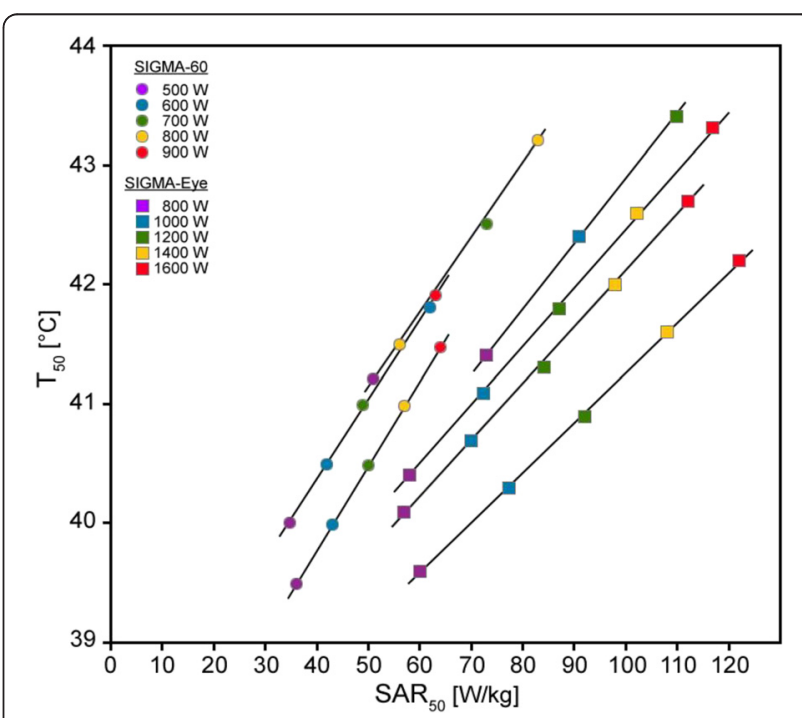

Fig. 3 Simulated characteristic lines in a SAR/temperature diagram for both applicators and the available patient models by variation of total power until a threshold temperature is exceeded. Classification in easy (lines shifted to the left) - and difficult-to-heat (lines shifted to the right) patients. As the curves were very similar for patients treated in the Sigma-60 applicator we show only 3 curves for this applicator (the remaining curves would lie in the interspace between the depicted curves) to enhance the clarity of the figure 


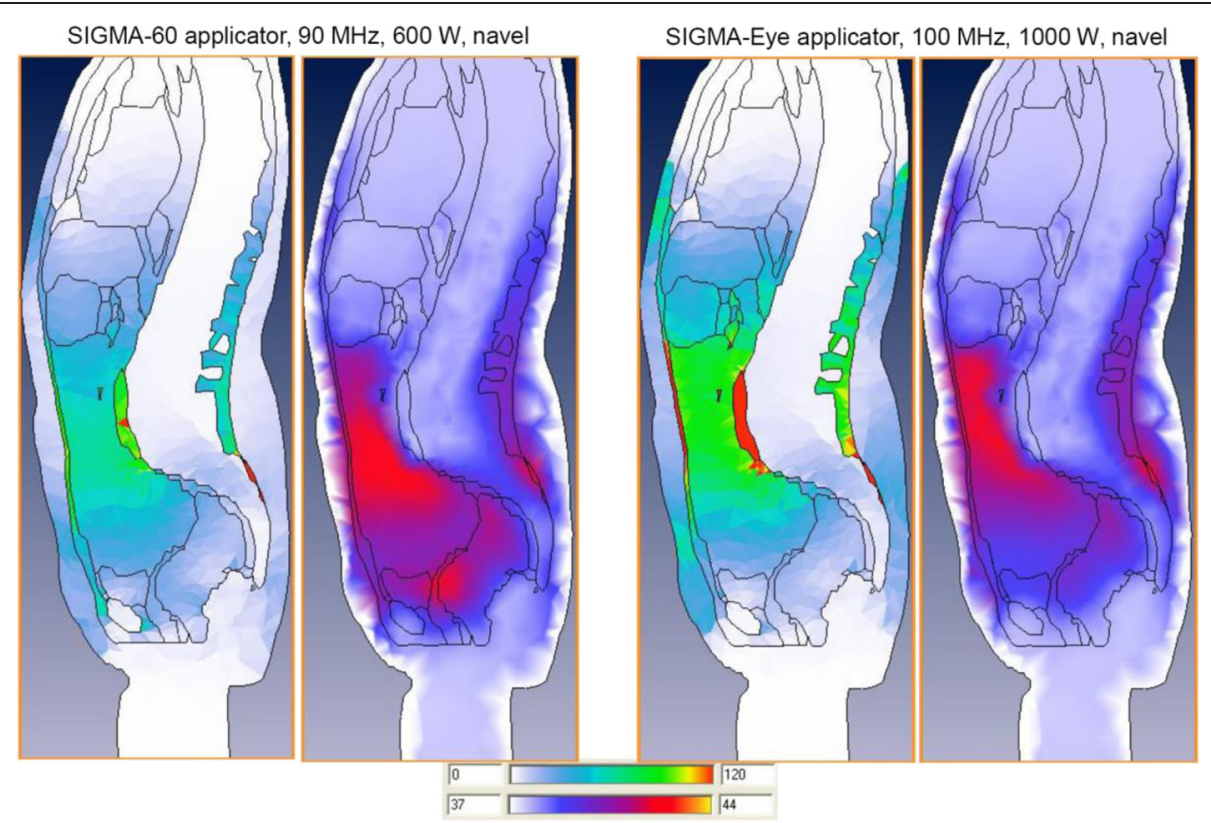

Fig. 4 Comparison of typical SAR and temperature patterns gained with the SIGMA-60 applicator (left) and the SIGMA-Eye applicator (right). In the SIGMA-60 the pelvic region is more effectively heated, while the upper abdomen is better covered in the SIGMA-Eye applicator. The dorsal temperature hotspot represents a local effect on the border of bone and fatty tissue with no consequence for nearby structures, particularly there is no critical temperature enhancement in the bony shielded spinal canal

$50^{\circ}$ at the outer rings was performed. The opportunity of proper phase control of SIGMA-Eye in comparison to SIGMA-60 and the resulting effects in effective heating volume are depicted in Figs. 5 and 6. Note that for the larger volume an elevation of the total power is also necessary in order to ensure a superior temperature distribution with higher $\mathrm{T}_{90}$.

\section{Discussion}

Our simulation study based on clinical experience and measurements suggests an appropriate abdominal heating capability using the annular-phased-array technique. Furthermore our results show the applicability of SIGMA-60 as well as SIGMA-Eye with a small advantage of SIGMAEye applicator in abdominal RHT.

CRS/HIPEC, with its notable treatment related toxicity and mortality rates, are objective of several ongoing and completed retrospective and prospective trials. As reported in retrospective and phase I/II trials there appears to be a benefit for patients with pseudomyxoma peritonei, peritoneal mesothelioma and colorectal cancer with PC $[5,6,20,21]$. However, for other cancer types e.g. ovarian cancer even less supportive data for CRS/HIPEC exist. Notably, even in experienced centers CRS/HIPEC procedures were associated with toxicity rates between 0 and $40 \%$ and mortality rates of $0-10 \%$ and only a limited number of patients (after careful selection) are actually

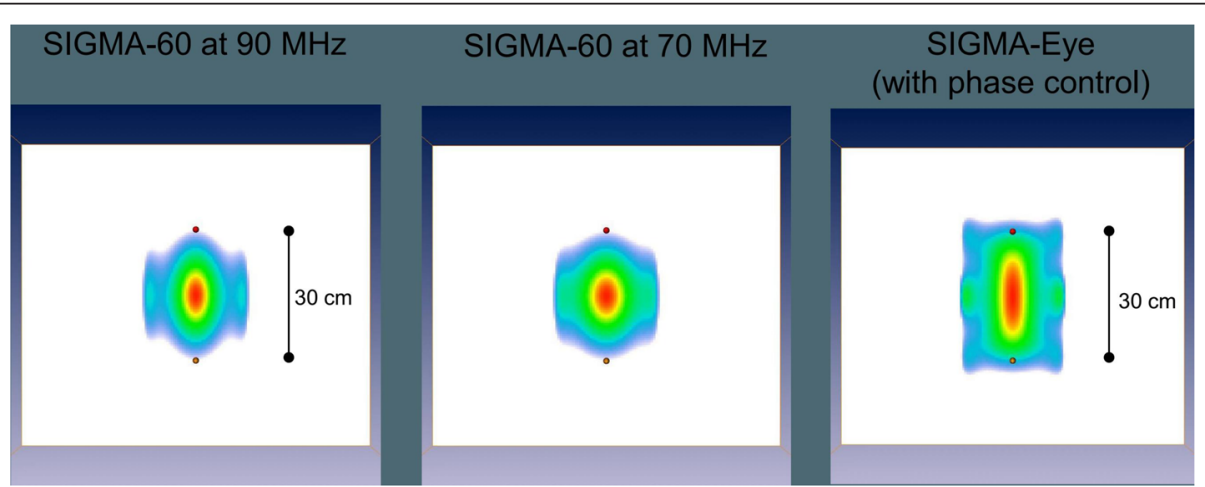

Fig. 5 Comparison of the pattern of effective heated abdominal volume for SIGMA-60 applicator (frequencies of $90 \mathrm{MHz}$ and $70 \mathrm{MHz}$ ) and the broadened pattern enabled by proper phase control in SIGMA-Eye applicator 


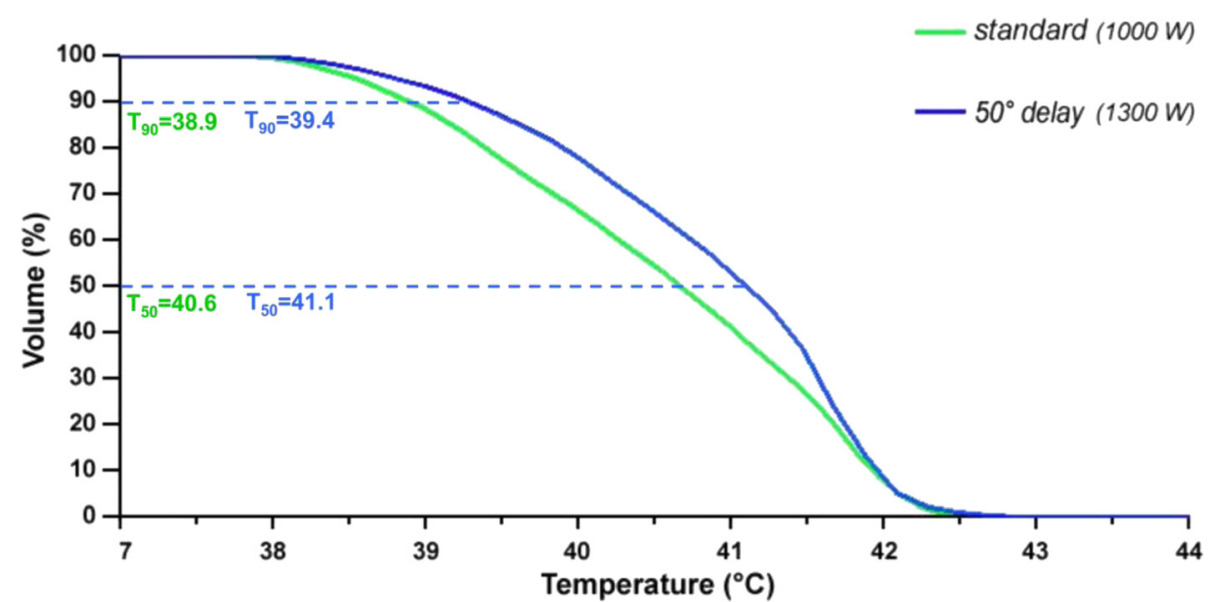

Fig. 6 Changes of effective heated volume using SIGMA-Eye applicator with standard adjustment and under proper phase control with a delay of $50^{\circ}$ on the outer applicator rings

eligible for CRS/HIPEC. In patients with PC of non gynecologic origin grade 3-4 toxicity rates range from 29 to $40 \%$, whereas in ovarian cancer PC patients in 0-32\% grade 3-4 morbidity was reported. Moreover in gynecologic patients grade 3-4 hematologic morbidity of $8-31 \%$ was observed due to application of the intraperitoneal chemotherapy [7-11]. Therefore, it appears unlikely that CRS/HIPEC will truly improve the therapeutic ratio for a large number of patients suffering from PC and thus, other preferably non-invasive and better tolerated approaches are needed to intensify the treatment of patients with PC.

Experiences with whole body hyperthermia (WBH) showed promising results in several Phase II studies treating colorectal, ovarian and other cancer subtypes. However, it is associated with a grade III/IV toxicity rate of $3.1 \%$ in 1300 described treatments. Moreover, the rate of deaths in the first 30 days after WBH plus chemotherapy treatment was $0.8 \%$. Furthermore WBH requires general anaesthesia or deep analgesic sedation and is therefore also logistically demanding. Clinical experience shows that WBH is limited to 3-6 sessions because of this burden. Therefore, WBH is neither for a palliative nor for a maintenance approach suitable [22-24].

In contrast RHT has been demonstrated as effective and is associated with a very low rate of treatment related toxicities [12-14]. But it is important to consider current quality assurance guidelines for treatment planning and application $[25,26]$. Moreover, with few exceptions (pace maker, severe cardiac disease, metal implants in the treatment area, acute thrombosis), RHT has less strict inclusion criteria and can potentially be applied to a larger proportion of patients suffering from PC than CRS/ HIPEC and WBH with its limitations as described before. So far no randomized trial was initiated to test the combination of RHT and chemotherapy vs. chemotherapy alone. Our group is currently developing a randomized phase II trial who will address this question in patients with recurrent ovarian cancer.

There are several limitations to our study. A major problem are direct (invasive) temperature measurements in the abdomen/peritoneum. However, there are substantial practical obstacles to perform invasive measurements in these seriously sick patients. Invasive thermometry would require a laparoscopic placement or at least a radiological intervention. Both procedures ar associated with morbidity and discomfort (and even risks of complications as bleeding or infection). In particular, invasive thermometry is not suitable for routine use. On the other hand, we widely used minimally invasive temperature measurements with endoluminal catheters in rectum, vagina and/or bladder. The replacement of invasive intra-tumoral measurements by endoluminal reference measurements (Fig. 1) is a generally applied method and has been extensively discussed elsewhere $[27,28]$. Here, we combined potent planning tools matched to reference measurements in order to achieve the information outlined in this paper. This might be an acceptable strategy for a first elucidation of abdominal heating. Abdominal heating has a wide spectrum of oncological indications (pancreatic cancer, ovarian cancer, colorectal cancer, gastric cancer and peritoneal carcinomatosis of different kinds), probably more than in case of pelvic heating. For a broad application of abdominal heating in the future, better specific quality parameters a strongly desirable. Noninvasive thermometry (MRI-thermometry) is the method of choice to obtain the information needed with low morbidity and clearly has much better acceptance in comparison to invasive and minimally invasive thermometry [29]. However until now, noninvasive MRI-thermometry in the abdomen is still a great challenge. Inhomogeneities and motion are great obstacles to implement noninvasive 
MRI-thermometry under routine conditions. For abdominal heating MRI-thermometry of the liver could be a promising concept, because it appears feasible and would provide the mean temperature of the target, i.e. abdomen. Another point of criticism might be the assumption of 20 $\mathrm{ml} / 100 \mathrm{~g} / \mathrm{min}$ for the abdominal perfusion which has been estimated considering current physiologic knowledge and in accordance with former studies [19]. In case of incorrect perfusion parameters the temperature distribution would change. For example a higher perfusion rate would lead to lower abdominal temperatures. Again, MRIthermometry will elucidate the behavior of abdominal perfusion under heating conditions, which is a complex and not yet completely resolved physiological issue. Despite these objections we believe that an appropriate abdominal heat distribution can be achieved by RHT and further studies with RHT in the future are warranted in patients with PC.

\section{Conclusion}

Our results suggest an adequate heating of the abdomen and therefore abdominal regional hyperthermia in PC patients appears feasible. This may facilitate the use of RHT in future multidisciplinary trials for the treatment of PC. To ensure a qualitative regional abdominal hyperthermia the combination of a hyperthermia treatment planning and non invasive MRI-thermometry should further be developed to be a standard combination in future treatments [25]. The SIGMA-Eye applicator appears to be the optimal applicator for abdominal RHT.

\section{Competing interests}

The authors declare that they have no competing interests.

\section{Authors' contributions}

$\mathrm{MB}$ and PW drafted the manuscript. PW, MB, PG and JN participated in the design of the study or made substantial contributions to acquisition, analysis, and interpretation of the data. MW, VB and JS provided critical review of the manuscript for important intellectual content. All authors read and approved the final manuscript.

\section{Acknowledgements}

We thank H. Ganter and J. Gellermann for her technical assistance and intellectual input.

\section{Author details \\ 'Department of Radiation Oncology, Charité Universitätsmedizin Berlin, Augustenburger Platz 1, 13353 Berlin, Germany. ${ }^{2}$ Charité Comprehensive Cancer Center, Charité Universitätsmedizin Berlin, Berlin, Germany. ${ }^{3}$ Department of Gynecology, Charité Universitätsmedizin Berlin, Berlin, Germany.}

Received: 19 March 2015 Accepted: 30 June 2015

Published online: 30 July 2015

\section{References}

1. Sadeghi B, Arvieux C, Glehen O, Beaujard AC, Rivoire M, Baulieux J, et al. Peritoneal carcinomatosis from non-gynecologic malignancies: results of the EVOCAPE 1 multicentric prospective study. Cancer. 2000;88(2):358-63.

2. Coccolini F, Gheza F, Lotti M, Virzi S, lusco D, Ghermandi C, et al. Peritoneal carcinomatosis. World J Gastroenterol. 2013;19(41):6979-94.
3. Sehouli J, Senyuva F, Fotopoulou C, Neumann U, Denkert C, Werner L, et al. Intra-abdominal tumor dissemination pattern and surgical outcome in 214 patients with primary ovarian cancer. J Surg Oncol. 2009;99(7):424-7.

4. Ozols RF. Treatment goals in ovarian cancer. Int J Gynecol Canc. 2005;15 Suppl 1:3-11.

5. Yan TD, Deraco M, Baratti D, Kusamura S, Elias D, Glehen O, et al. Cytoreductive surgery and hyperthermic intraperitoneal chemotherapy for malignant peritoneal mesothelioma: multi-institutional experience. J Clin Oncol. 2009;27(36):6237-42

6. Verwaal VJ, van Ruth $\mathrm{S}$, de Bree $\mathrm{E}$, van Sloothen GW, van Tinteren $H$, Boot $H$, et al. Randomized trial of cytoreduction and hyperthermic intraperitoneal chemotherapy versus systemic chemotherapy and palliative surgery in patients with peritoneal carcinomatosis of colorectal cancer. J Clin Oncol. 2003;21(20):3737-43.

7. Ahmed S, Stewart JH, Shen P, Votanopoulos KI, Levine EA. Outcomes with cytoreductive surgery and HIPEC for peritoneal metastasis. J Surg Oncol. 2014;110(5):575-84.

8. Elias D, Goere D, Dumont F, Honore C, Dartigues P, Stoclin A, et al. Role of hyperthermic intraoperative peritoneal chemotherapy in the management of peritoneal metastases. Eur J Cancer. 2014;50(2):332-40.

9. Bakrin N, Classe JM, Pomel C, Gouy S, Chene G, Glehen O. Hyperthermic intraperitoneal chemotherapy (HIPEC) in ovarian cancer. J Visc Surg. 2014;151(5):347-53.

10. Chua TC, Robertson G, Liauw W, Farrell R, Yan TD, Morris DL. Intraoperative hyperthermic intraperitoneal chemotherapy after cytoreductive surgery in ovarian cancer peritoneal carcinomatosis: systematic review of current results. J Cancer Res Clin Oncol. 2009;135(12):1637-45.

11. Chiva LM, Gonzalez-Martin A. A critical appraisal of hyperthermic intraperitoneal chemotherapy (HIPEC) in the treatment of advanced and recurrent ovarian cancer. Gynecol Oncol. 2015;136(1):130-5.

12. Wust P, Hildebrandt B, Sreenivasa G, Rau B, Gellermann J, Riess H, et al. Hyperthermia in combined treatment of cancer. Lancet Oncol. 2002;3(8):487-97.

13. Cho C, Wust P, Hildebrandt B, Issels RD, Sehouli J, Kerner T, et al. Regional hyperthermia of the abdomen in conjunction with chemotherapy for peritoneal carcinomatosis: evaluation of two annular-phased-array applicators. Int J Hyperther. 2008;24(5):399-408.

14. Fotopoulou C, Cho CH, Kraetschell R, Gellermann J, Wust P, Lichtenegger W, et al. Regional abdominal hyperthermia combined with systemic chemotherapy for the treatment of patients with ovarian cancer relapse: Results of a pilot study. Int J Hyperther. 2010;26(2):118-26.

15. Sreenivasa G, Gellermann J, Rau B, Nadobny J, Schlag P, Deuflhard P, et al. Clinical use of the hyperthermia treatment planning system HyperPlan to predict effectiveness and toxicity. Int J Radiat Oncol Biol Phys. 2003;55(2):407-19.

16. Gellermann J, Wust P, Stalling D, Seebass M, Nadobny J, Beck R, et al. Clinical evaluation and verification of the hyperthermia treatment planning system hyperplan. Int J Radiat Oncol Biol Phys. 2000;47(4):1145-56.

17. Seebass M, Beck R, Gellermann J, Nadobny J, Wust P. Electromagnetic phased arrays for regional hyperthermia: optimal frequency and antenna arrangement. Int J Hyperther. 2001;17(4):321-36.

18. Gabriel C, Gabriel S, Corthout E. The dielectric properties of biological tissues: I. Literature survey. Phys Med Biol. 1996;41(11):2231-49.

19. Wust P, Stahl H, Loffel J, Seebass M, Riess H, Felix R. Clinical, physiological and anatomical determinants for radiofrequency hyperthermia. Int J Hyperther. 1995;11(2):151-67.

20. Chua TC, Moran BJ, Sugarbaker PH, Levine EA, Glehen O, Gilly FN, et al. Early- and long-term outcome data of patients with pseudomyxoma peritonei from appendiceal origin treated by a strategy of cytoreductive surgery and hyperthermic intraperitoneal chemotherapy. J Clin Oncol. 2012;30(20):2449-56.

21. Sugarbaker PH, Ryan DP. Cytoreductive surgery plus hyperthermic perioperative chemotherapy to treat peritoneal metastases from colorectal cancer: standard of care or an experimental approach? Lancet Oncol. 2012;13(8):e362-9.

22. Hegewisch-Becker S, Gruber Y, Corovic A, Pichlmeier U, Atanackovic D, Nierhaus $A$, et al. Whole-body hyperthermia (41.8 degrees C) combined with bimonthly oxaliplatin, high-dose leucovorin and 5-fluorouracil 48-hour continuous infusion in pretreated metastatic colorectal cancer: a phase II study. Ann Oncol. 2002;13(8):1197-204.

23. Hildebrandt B, Hegewisch-Becker S, Kerner T, Nierhaus A, Bakhshandeh-Bath A, Janni W, et al. Current status of radiant whole-body hyperthermia at 
temperatures $>41.5$ degrees $C$ and practical guidelines for the treatment of adults. The German 'Interdisciplinary Working Group on Hyperthermia'. Int J Hyperthermia. 2005;21(2):169-83.

24. Hildebrandt B, Drager J, Kerner T, Deja M, Loffel J, Stroszczynski C, et al. Whole-body hyperthermia in the scope of von Ardenne's systemic cancer multistep therapy (sCMT) combined with chemotherapy in patients with metastatic colorectal cancer: a phase I/II study. Int J Hyperthermia. 2004;20(3):317-33.

25. Kok P. Current state of the art regional hyperthermia planning (multi-institutional article). Radiat Oncol. 2015. (in press).

26. van Rhoon $\mathrm{G}$. Why high quality hyperthermia is important, lessons to be learned (multi-institutional article). Radiat Oncol. 2015. (in press).

27. Wust P, Gellermann J, Harder C, Tilly W, Rau B, Dinges S, et al. Rationale for using invasive thermometry for regional hyperthermia of pelvic tumors. Int J Radiat Oncol Biol Phys. 1998;41(5):1129-37.

28. van der Zee J, Peer-Valstar JN, Rietveld PJ, de Graaf-Strukowska L, van Rhoon GC. Practical limitations of interstitial thermometry during deep hyperthermia. Int J Radiat Oncol Biol Phys. 1998;40(5):1205-12.

29. Wust $\mathrm{P}$, Cho CH, Hildebrandt B, Gellermann J. Thermal monitoring: invasive, minimal-invasive and non-invasive approaches. Int J Hyperthermia. 2006;22(3):255-62

\section{Submit your next manuscript to BioMed Central and take full advantage of:}

- Convenient online submission

- Thorough peer review

- No space constraints or color figure charges

- Immediate publication on acceptance

- Inclusion in PubMed, CAS, Scopus and Google Scholar

- Research which is freely available for redistribution 